\title{
Pathological Complete Response to Lenvatinib after Failure of Atezolizumab plus Bevacizumab in Unresectable Hepatocellular Carcinoma
}

\author{
Yusuke Johira $^{\text {a }}$ Tomokazu Kawaoka $^{\text {a }}$ Masanari Kosaka ${ }^{a}$ Yuki Shirane $^{\text {a }}$ Ryoichi Miura $^{a}$ \\ Serami Murakami ${ }^{a}$ Shigeki Yano ${ }^{a}$ Kei Amioka ${ }^{a}$ Kensuke Naruto ${ }^{a}$ Yuwa Ando ${ }^{a}$ \\ Yumi Kosaka $^{\text {a Kenichiro Kodama }}{ }^{a}$ Shinsuke Uchikawa ${ }^{a}$ Hatsue Fujino ${ }^{a}$ Atsushi Ono $^{a}$

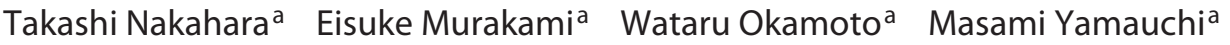 \\ Michio Imamura ${ }^{a}$ Kazuhiro Sentani ${ }^{b}$ Naohide Oue ${ }^{b}$ Koji Arihiro ${ }^{c}$ Shintaro Kuroda ${ }^{d}$ \\ Tsuyoshi Kobayashid $^{d}$ Hideki Ohdan $^{d}$ Kazuaki Chayama $^{a}$ Hiroshi Aikata $^{a}$ \\ aDepartment of Gastroenterology and Metabolism, Hiroshima University Hospital, Hiroshima, Japan; ${ }^{b}$ Department \\ of Molecular Pathology, Graduate School of Biomedical and Health Sciences, Hiroshima University, Hiroshima, \\ Japan; 'Department of Anatomical Pathology, Hiroshima University Hospital, Hiroshima, Japan; ${ }^{d}$ Department of \\ Gastroenterological Surgery, Hiroshima University Hospital, Hiroshima, Japan
}

Dear Editor,

The patient was a 56-year-old woman with cirrhosis caused by hepatitis $\mathrm{C}$ virus. Blood test results showed preserved liver function, indicating Child-Pugh class A and modified albumin-bilirubin (mALBI) grade 1, but the a-fetoprotein (AFP) level was significantly increased to 10,530 ng/mL (normal range: $0-10 \mathrm{ng} / \mathrm{mL}$ ). Dynamic computed tomography (CT) showed a hepatic mass, 40 $\mathrm{mm}$ in diameter, in the lateral segment of the liver, with strong and homogeneous enhancement in the arterial phase (shown in Fig. 1a). It presented hyperintensity in the hepatobiliary phase of gadolinium ethoxybenzyl diethylenetriaminepentaacetic acid-enhanced magnetic resonance imaging (EOB-MRI) (shown in Fig. 1b). Positron emission tomography showed marked fluorodeoxyglucose uptake by the tumor (shown in Fig. 1c). There was no intrahepatic or extrahepatic metastasis, but the lateral branch of the portal vein could not be detected, suggesting portal vein tumor thrombosis.

On diagnosis of unresectable hepatocellular carcinoma (HCC), considering high postoperative recurrence

karger@karger.com www.karger.com/lic

Karger"
C 2021 The Author(s).

Published by S. Karger AG, Basel

This is an Open Access article licensed under the Creative Commons Attribution-NonCommercial-4.0 International License (CC BY-NC) (http://www.karger.com/Services/OpenAccessLicense), applicable to the online version of the article only. Usage and distribution for commercial purposes requires written permission. rate of HCC with portal vein invasion if resected without preceding treatment, atezolizumab and bevacizumab were initiated at $1,200 \mathrm{mg}$ and $15 \mathrm{mg} / \mathrm{kg}$, respectively. However, CT after the second course revealed an increase in tumor size ( $80 \mathrm{~mm}$ in diameter) (shown in Fig. 1d); AFP level also increased rapidly (shown in Fig. 2). Therefore, lenvatinib was initiated as the second-line therapy at a dose of $8 \mathrm{mg} /$ day. Some adverse events induced by lenvatinib, such as hand-foot syndrome and thrombocytopenia, were observed. In particular, thrombocytopenia with a platelet count $<50,000 / \mu \mathrm{L}$ required temporary interruption and dose reduction to $4 \mathrm{mg} /$ day or $4 \mathrm{mg}$ and 8 $\mathrm{mg}$ on alternate days when lenvatinib was restarted. With this therapy, AFP level decreased immediately, and CT 4 weeks after initiation revealed remarkable tumor shrinkage accompanied by decreased tumor vascularity and viability; the tumor size decreased by $50 \%$, suggesting partial response on modified RECIST (shown in Fig. 1e). Liver function was not impaired by atezolizumab plus bevacizumab and lenvatinib, indicating Child-Pugh class $A$ and $\mathrm{mALBI}$ grade 1 . Considering that the tumor was 

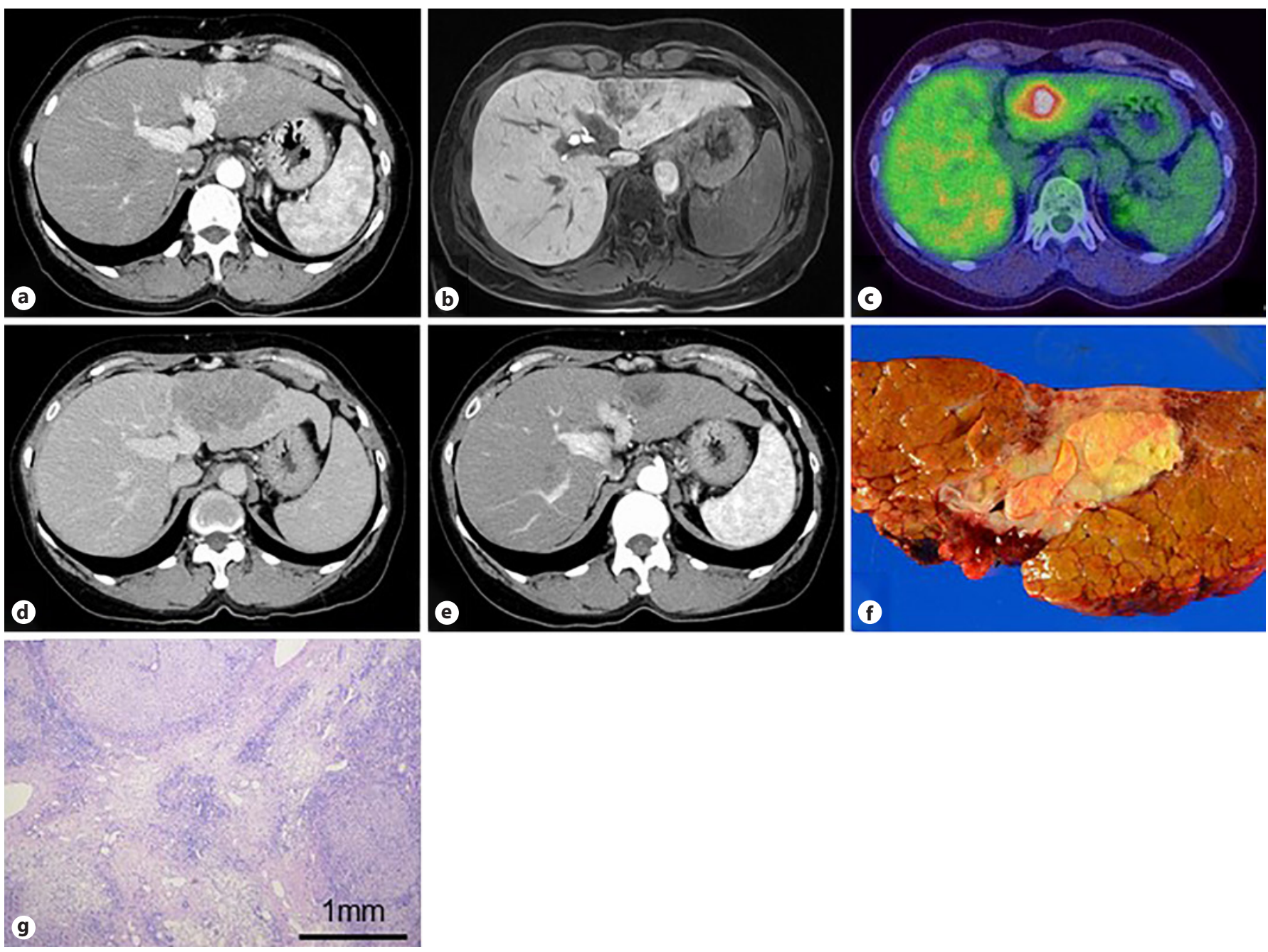

Fig. 1. a Dynamic CT arterial phase at the time of diagnosis. A tumor with strong and homogeneous enhancement was located in the lateral segment of the liver. $\mathbf{b}$ EOB-MRI at the time of diagnosis. The tumor was heterogeneously enhanced in the hepatobiliary phase. c FDG-PET at the time of diagnosis. The tumor showed marked FDG uptake. d Dynamic CT at late phase after the second course of atezolizumab plus bevacizumab. Tumor size significant-

well controlled and that the liver function was good, we performed conversion hepatectomy 13 weeks after initiating lenvatinib. A yellowish-white tumor, $45 \mathrm{~mm}$ in diameter, was observed macroscopically (shown in Fig. 1f). Microscopically, the tumor was totally necrotic with no viable cells, and there was no vascular invasion (shown in Fig. 1g). No recurrence since 3 months postoperatively. To the best of our knowledge, this is the first case of pathological complete response of unresectable HCC to treatment with lenvatinib after failure of atezolizumab plus bevacizumab.

ly increased. e Dynamic CT arterial phase 4 weeks after lenvatinib initiation. The size and vascularity of the tumor were significantly decreased. $\mathbf{f}$ Macroscopic findings on surgical specimen. $\mathbf{g}$ Microscopic findings on surgical specimen. The tumor was completely necrotic, with no viable tumor cells. CT, computed tomography; EOB-MRI, gadolinium ethoxybenzyl diethylenetriaminepentaacetic acid-enhanced magnetic resonance imaging.

HCCs are classified into 3 classes based on the immune status of the microenvironment. Among the 3 classes, the "immune exclusion class," accounting for around 30\% of cases, is characterized by activation of $\beta$-catenin induced by CTNNB1 mutation and tends to be resistant to treatment with immune checkpoint inhibitors. Ueno et al. [1] showed that $\mathrm{Wnt} / \beta$-catenin signaling induces the expression of organic anion-transporting polypeptide $1 \mathrm{~B} 3$, suggesting HCC enhancement in the hepatobiliary phase in EOB-MRI is driven by CTNNB1 mutation. In our patient, considering that the lesion was also enhanced in the

Liver Cancer 2022;11:174-177 


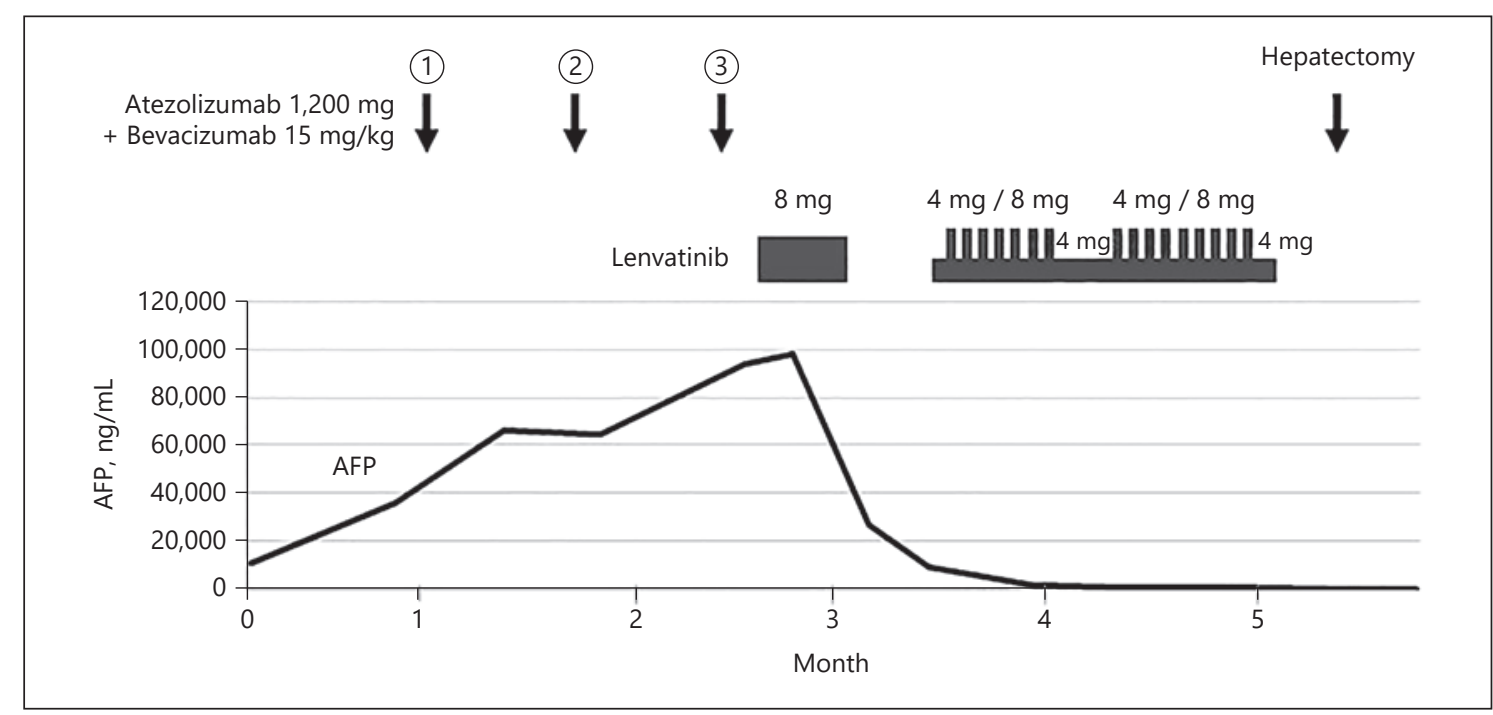

Fig. 2. We started atezolizumab plus bevacizumab for a single, intrahepatic, unresectable HCC due to portal vein tumor thrombosis. However, CT after the second course revealed a remarkable increase in the tumor size, and AFP level also increased rapidly, which led us to complete atezolizumab plus bevacizumab in 3 courses and initiate lenvatinib as the second-line therapy. Despite thrombocytopenia induced by lenvatinib, requiring temporary interruption and dose reduction, the response to lenvatinib was good, with the AFP level significantly decreased. CT performed 4

phase with a relative enhancement ratio of 0.99 , it might have been immune exclusion HCC driven by CTNNB1 mutation; this may explain the poor effect of atezolizumab plus bevacizumab.

Although homogeneously enhanced HCC in dynamic CT arterial phase treated with lenvatinib has a lower early objective response rate than heterogeneously enhanced HCC [2], our case represented a remarkable response to lenvatinib. One of the reasons may be that the response to lenvatinib in iso-high intensity HCC in the hepatobiliary phase of EOB-MRI was not diminished like that in atezolizumab plus bevacizumab, which was reported by Kubo et al. [3]. Second, it has been reported that lenvatinib was initiated immediately after the failure of anti-PD-1/PD-L1 therapy, which is expected to have a more potent antitumor effect due to synergism than when initiated alone as first-line therapy [4]. This is supported by the fact that anti-PD-1 antibody binds to PD- 1 for $>20$ weeks.

Moreover, Yamauchi et al. [5] showed that FGFR4 expression is higher in $\mathrm{HCC}$ with activated $\mathrm{Wnt} / \beta$-catenin signaling and that HCC with FGFR4 expression has a significantly better response rate among patients with advanced HCC treated with lenvatinib. Our case could be driven by a CTNNB1 mutation leading to high FGFR4 weeks after lenvatinib initiation revealed remarkable tumor shrinkage accompanied by decreased tumor vascularity and viability, suggesting partial response on modified RECIST. Liver function was not impaired, indicating Child-Pugh class A and mALBI grade 1 . We performed conversion hepatectomy 13 weeks after the initiation of lenvatinib. HCC, hepatocellular carcinoma; CT, computed tomography; AFP, a-fetoprotein; mALBI, modified albumin-bilirubin.

expression. Hence, regardless of previous therapy, treatment with lenvatinib would be appropriate.

Various treatment options are currently available for unresectable HCC. It is important to be aware of the presence of cases primarily resistant to atezolizumab plus bevacizumab and consider alternative therapy in such cases.

\section{Conflict of Interest Statement}

The authors have no conflicts of interest to declare.

\section{Funding Sources}

Not applicable.

\section{Author Contributions}

T.K. and H.A. contributed to pretreatment diagnosis and systemic treatment. S.K., T.K., and H.O. performed the operation. K.S., N.O., and K.A. carried out the pathological diagnosis. Y.J. wrote the manuscript. All authors have read and approved the final version of the manuscript, and all authors fulfill the COPE (Committee on Publication Ethics) requirements for authorship. 


\section{References}

1 Ueno A, Masugi Y, Yamazaki K, Komuta M, Effendi K, Tanami Y, et al. OATP1B3 expression is strongly associated with $\mathrm{Wnt} / \beta$-catenin signalling and represents the transporter of gadoxetic acid in hepatocellular carcinoma. J Hepatol. 2014 Nov;61(5):1080-7.

2 Kawamura Y, Kobayashi M, Shindoh J, Kobayashi Y, Kasuya K, Sano T, et al. Pretreatment heterogeneous enhancement pattern of hepatocellular carcinoma may be a useful new predictor of early response to lenvatinib and overall prognosis. Liver Cancer. 2020 Jun; 9(3):275-92.

3 Kubo A, Suda G, Kimura M, Maehara O, Tokuchi Y, Kitagataya T, et al. Characteristics and lenvatinib treatment response of unresectable hepatocellular carcinoma with iso-high intensity in the hepatobiliary phase of EOB-MRI. Cancers. 2021 Jul;13(14): 3633.

4 Aoki T, Kudo M, Ueshima K, Morita M, Chishina $\mathrm{H}$, Takita $\mathrm{M}$, et al. Exploratory analysis of lenvatinib therapy in patients with unresectable hepatocellular carcinoma who have failed prior PD-1/PD-L1 checkpoint blockade. Cancers. 2020 Oct;12(10):3048.

5 Yamauchi M, Ono A, Ishikawa A, Kodama $\mathrm{K}$, Uchikawa S, Hatooka H, et al. Tumor fibroblast growth factor receptor 4 level predicts the efficacy of lenvatinib in patients with advanced hepatocellular carcinoma. Clin Transl Gastroenterol. 2020 May;11(5): e00179. 